\title{
Profiling of Hepatocellular Carcinoma Cell Cycle Regulating Genes Targeted by Calycosin
}

\author{
Dongqing Zhang, ${ }^{1}$ Shufang Wang, ${ }^{1}$ Liguo Zhu, ${ }^{1}$ Yaping Tian, ${ }^{1}$ Haibao Wang, \\ Yuan Zhuang, ${ }^{1}$ Yu Li, ${ }^{2}$ and Deqing Wang ${ }^{1}$ \\ ${ }^{1}$ Department of Blood Transfusion, General Hospital of PLA, Beijing 100853, China \\ ${ }^{2}$ Department of Toxicology, Beijing Center for Diseases Control and Prevention, Beijing 100013, China
}

Correspondence should be addressed to Yu Li; liyu_tg@yahoo.com.cn and Deqing Wang; deqingw@vip.sina.com

Received 30 July 2013; Revised 18 October 2013; Accepted 2 December 2013

Academic Editor: Wei Mike Liu

Copyright (C) 2013 Dongqing Zhang et al. This is an open access article distributed under the Creative Commons Attribution License, which permits unrestricted use, distribution, and reproduction in any medium, provided the original work is properly cited.

\begin{abstract}
We cocultured calycosin with human hepatocellular carcinoma cell line (BEL-7402) to investigate the effect on cell proliferation. Calycosin can markedly block the cell growth in $\mathrm{G}_{1}$ phase $(P<0.01)$ on the $\mathrm{IC}_{50}$ concentration. There were seventeen genes involved in cell-cycle regulation showing differentially expressed in treated cells detected by gene chip. Eight genes were upregulated and nine genes were downregulated. Downregulated TFDP-1, CDKN2D, and SPK2 and upregulated CDC2 and CCNB1 might affect cell cycle of tumor cells. Furthermore, we checked the transcription pattern using 2D gel method to find different expression of proteins in human hepatocellular carcinoma cells after exposure to calycosin. Fourteen proteins were identified by matrix-assisted laser desorption/ionization-time of flight-mass spectrometry (MALDI-TOF-MS). Twelve proteins expression were increased such as transgelin 2, pyridoxine $5^{\prime}$-phosphate, stress-induced-phosphoprotein 1 , peroxiredoxin 1 , endoplasmic reticulum protein 29 , and phosphoglycerate mutase 1 . Only thioredoxin peroxidase and high-mobility group boxl proteins' expression decreased. Both genes and proteins changes might be relate to the mechanism of antitumor effect under treatment of calycosin. In conclusion, calycosin has a potential effect to inhibit the BEL-7402 cell growth by inhibiting some oncogene expression and increasing anticancer genes expression, what is more, by blocking cell cycle.
\end{abstract}

\section{Introduction}

Astragalus mongholicus can improve body's specific and nonspecific immune functions, promote immunocytokine generating, and inhibit the growth of tumor cells. Astragalus mongholicus enhances immune function by increasing the activity of certain white blood cells which increases the production of antibodies (IgA and IgG), increases the production of interferon, and stimulates natural killer cells.

In addition to boosting immunity, Astragalus has antibacterial, anti-inflammatory, and antiviral effects. It contains numerous components, including polysaccharides, flavonoids, triterpene glycosides, amino acids, and trace minerals. Besides the main function of enhancing the NK cells activity, a series of studies also indicated the remarkable inhibition of flavonoids of Astragalus (TFA) on human hepatoma cell line BEL-7402 and a variety of leukemia cells proliferation in vitro
$[1,2]$, and $\mathrm{Xu} \mathrm{Du}$-juan found that Astragalus significantly inhibited the growth of transplanted hepatoma (HepA) in mice and sarcoma (S180) and the growth of HeLa cells in vitro [3]. These studies denoted that the ingredients in Astragalus not only had the antitumor effect on the overall level but also had direct inhibition on tumor cell in vitro. Reported TFA also blocked the cell-cycle in $G_{0} / G_{1}$ phase, after incubating HeLa cells together for 5 days [3].

However, there were no reports about the inhibitory action of calycosin, one of the capital chemical compositions of TFA on tumor cells and the mechanism of anti-tumor. The calycosin was a main active ingredient of the Astragalus and liquorice [1]. In this study, neutral red (NR) assay, flow cytometry, gene chip detection, and two-dimensional gel electrophoresis were performed to investigate the effect of calycosin on human hepatocellular carcinoma cell line (BEL-7402), in an attempt discover some detailed mechanism. We found that 
calycosin can hinder BEL-7402 cells into S phase and inhibit tumor cell proliferating rapidly by blockage in $G_{1}$ phase.

\section{Materials and Methods}

2.1. Materials. Calycosin was isolated and identified by the Department of Biochemistry of Chinese People's Liberation Army (PLA) General Hospital. The kits of cell-cycle were offered by the company of BD. The gene chips of human cellcycle were prepared and analyzed by the CapitalBio Corporation.

2.2. Culture of BEL-7402 Cells. Human hepatocellular carcinoma cell line (BEL-7402) was obtained from the Chinese Academy of Science Shanghai Institute of Cell Biology. BEL7402 cells were cultured in RPMI1640 medium (GIBCO) containing penicillin and streptomycin and 10\% inactivated fetal bovine serum under the conditions of $37^{\circ} \mathrm{C}, 5 \% \mathrm{CO}_{2}$. When the cells were at the stage of logarithm growth, all the experiments were carried out.

2.3. Determination of the $50 \%$ Inhibitory Concentration $\left(I C_{50}\right)$ of Calycosin. The $\mathrm{IC}_{50}$ was measured by neutral red (NR) assay. The BEL-7402 cells in the stage of logarithm growth were added into 96-culture plate at the density of $1 \times 10^{4}$ per well in $0.1 \mathrm{~mL}$ volume. Different concentrations of drugs were added (final concentrations were 0.004, 0.011, 0.035, 0.111, 0.352 , and $1.113 \mathrm{mmol} / \mathrm{L}$ ) after 24 hours of incubation, four duplicates in each group. After 24 hours of incubation, neutral red was added to a final concentration $50 \mathrm{mg} / \mathrm{L}$. Staining solution was discarded after of being incubated for 2 hours under the condition of $37^{\circ} \mathrm{C}$. Then to each well was added $100 \mu \mathrm{L}$ extract solution (volume fraction of $1 \%$ the acetic acid and 50\% ethanol), settled at room temperature for 20 minutes. OD was determined in the wavelength of $570 \mathrm{~nm}$ by the ELISA instrument and the growth inhibited rate of cancer cells was calculated.

2.4. Determination of Cell-Cycle by Flow Cytometry. Each $25 \mathrm{~cm}^{2}$ culture flask was plated with cells at the density of $7.5 \times 10^{5}$. Drugs were added at the concentration of the $\mathrm{IC}_{50}$. After 24 hours the cells were digested by trypsin and washed twice by PBS. Precooled $70 \%$ ethanol was then added to start fixation for at least 12 hours at $4^{\circ} \mathrm{C}$. Fixed cells were washed twice by PBS. RNaseA was then added to the final concentration of $50 \mu \mathrm{g} / \mathrm{mL}$ and incubated at $37^{\circ} \mathrm{C}$ for 30 minutes. Propidium iodide (PI) was added and the labeled cells were loaded to Model ABC flow cytometry analyzer for analysis.

2.5. Preparation of RNA. Total RNA was prepared from control cells and treated BEL-7402 cells using Trizol reagent. Cells were washed twice by PBS; then Trizol reagent was added ( $1 \mathrm{ml}$ Trizol for $5-10 \times 10^{6}$ cell) to extract the RNA according to the standard operation. RNA was concentrated by the precipitation method of isopropyl alcohol, purified by the NucleoSpin RNA clean-up kit. The OD of the RNA was measured by the spectrophotometer and the quality of the RNA was checked by the denaturing formaldehyde gel electrophoresis.

2.6. Determination of Gene Expression of Cell-Cycle. The samples were labeled with fluorescence by the method of RNA labeling and then were air-dried after being purified. The labeled samples were dissolved in hybridization solution and hybridized at $42^{\circ} \mathrm{C}$ overnight. The samples were washed in the washing solutions 1 , followed by washing solution 2 . Hybridized gene chips were scanned after being dried using dual-channel laser scanner of LuxScan $10 \mathrm{~K} / \mathrm{A}$ (CapitalBio Corporation). The images of gene chip were analyzed by image analysis software of CapitalBio (CapitalBio Corporation). Then the signal of pictures was changed into digital signal. The data in the gene chip were normalized by the Lowess method. At last, the differentially expressed genes were determined by the one-class method of SAM. The test was repeated twice.

2.7. Two-Dimensional Gel Electrophoresis and Collection and Analysis of Gel Images. The BEL-4702 cells were divided into two groups, one group which was treated with calycosin at the concentration of $\mathrm{IC}_{50}$ and the blank. Cell proteins were separated by 2DE method and then silver-stained.

Two-dimensional electrophoresis gel after being stained was scanned using gel-image scanner (Amersham Pharmacia Biotech Company) and digital image document was analyzed by software (Image Master 2D Elite 5.0) for obtaining the relative molecular mass $\mathrm{Mr}, \mathrm{pI}$, isoelectric point $\mathrm{pI}$, and relative amount of proteins.

2.8. Mass Spectrum Identification of Differentially Expressed Protein Points. Protein dose for two-dimensional gel electrophoresis was added to $1 \mathrm{mg}$ and then stained. Decoloration, trypsin enzymolysis in gel, and extraction of peptide were proceeded on after some differential protein points were excised; last peptide mass fingerprint spectrum (PMF) was analyzed using MALDI-TOF-MS. Evaluation of results reliability depended on the matching rate of peptides and sequence coverage rate of matched peptides on corresponding proteins.

2.9. Statistical Method. SPSS15.0 software was used for analysis $\mathrm{IC}_{50}$ was calculated by pro method (Probit). The statistical discrepancy between cell-cycles was examined by $t$-test. Cluster 3.0 software was used for gene expression, the method of hierarchical and average linkage for cluster analysis. Differential expression genes were analyzed for statistical significance on MAS (molecule annotation system).

\section{Results}

3.1. Examination $I C_{50}$ of Calycosin on BEL-7402 Cell. The BEL-7402 cells were treated with calycosin for 24 hours in different dosages of calycosin as shown in Table 1. The inhibition of calycosin on hepatocellular carcinoma cell at concentration of between 0.035 and $1.113 \mathrm{mmol} / \mathrm{L}$ was shown 
TABLE 1: The effect of calycosin on proliferation of BEL-7402 cells $(\bar{x} \pm s)$.

\begin{tabular}{lcc}
\hline $\begin{array}{l}\text { Calycosin concentration } \\
(\mathrm{mmol} / \mathrm{L})\end{array}$ & OD value & Inhibition rate \% \\
\hline Control (0.5\% DMSO) & $0.421 \pm 0.045$ & 0.5 \\
0.004 & $0.410 \pm 0.036$ & 3.1 \\
0.111 & $0.306 \pm 0.013^{* *}$ & 27.7 \\
0.352 & $0.156 \pm 0.042^{* *}$ & 63.1 \\
1.113 & $0.084 \pm 0.021^{* *}$ & 80.1
\end{tabular}

Data expressed as mean $\bar{x} \pm s,{ }^{* *} P<0.01$ compared with control.

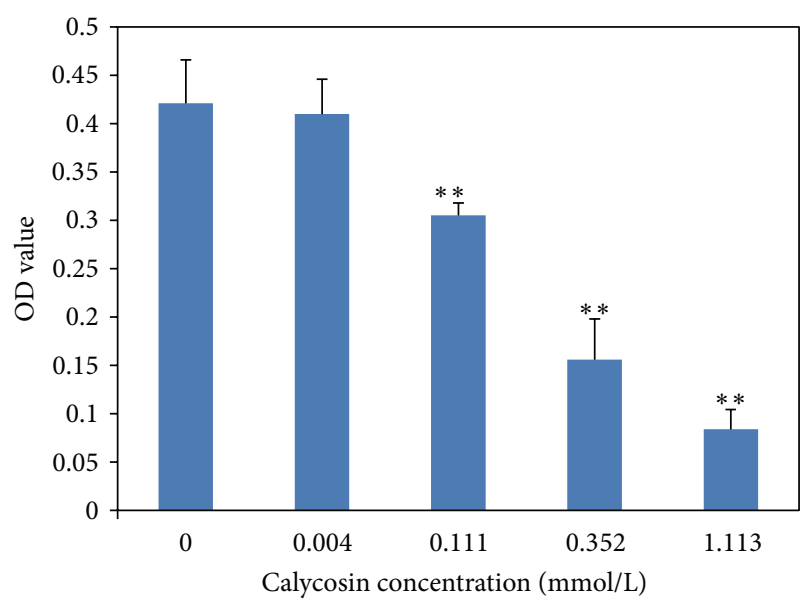

FIGURE 1: The effect of calycosin on proliferation of BEL-7402 cells.

as the significant dose-effect relationship. After the effect of calycosin on BEL-7402 for 24 hours, $\mathrm{IC}_{50}$ was $0.246 \mathrm{mmol} / \mathrm{L}$ by Probit analysis and the $95 \%$ confidence interval was 0.189 $0.336 \mathrm{mmol} / \mathrm{L}$ (Figure 1).

Different concentrations of calycosin were added (final concentrations were $0.004,0.011,0.035,0.111,0.352$, and $1.113 \mathrm{mmol} / \mathrm{L}$ ), four duplicates every group, including blank and solvent control (0.5\% DMSO). It shows that calycosin has a significant inhibitory effect on human hepatocellular carcinoma BEL-7402 cell in vitro. ${ }^{* *}$ means that $P<0.01$ compared with control.

3.2. Calycosin Reduces the Proliferation of BEL-7402 Cells. Calycosin was added at the concentration of the $\mathrm{IC}_{50}$. After 24 hours the cells were digested by trypsin and washed twice by PBS. The labeled cells were loaded to Model ABC flow cytometry analyzer for analysis The calycosin could block the cell growth cycle in $\mathrm{G}_{1}$ phase significantly comparing with control group (Figure 2) causing remarkable decrease of $S$ phase (Table 2).

3.3. The Effect of Calycosin on Gene Expression of CellCycle and Check Points. The A260/A280 value of total RNA extracted from samples was between 1.60 and 1.75 . In 10\% agarose gel electrophoresis, the bands of $18 \mathrm{~S}$ rRNA and $28 \mathrm{~S}$ rRNA were clear and the band of $28 \mathrm{~S}$ was the double of
TABLE 2: The effect of calycosin on cell cycle of BEL-7402 cells $(\bar{x} \pm s)$.

\begin{tabular}{lccc}
\hline Group & $\mathrm{G}_{1}$ Stage $(\%)$ & S Stage $(\%)$ & $\mathrm{G}_{2} / \mathrm{M}$ Stage $(\%)$ \\
\hline $\begin{array}{l}\text { Control } \\
(0.5 \% \text { DMSO) }\end{array}$ & $63.31 \pm 0.99$ & $28.84 \pm 0.82$ & $7.86 \pm 0.67$ \\
Calycosin & $74.27 \pm 2.31^{* *}$ & $12.94 \pm 4.53^{* *}$ & $12.79 \pm 2.28^{* *}$ \\
\hline Data expressed as mean $\bar{x} \pm s,{ }^{* *}$ means $P<0.01$ compared with control.
\end{tabular}

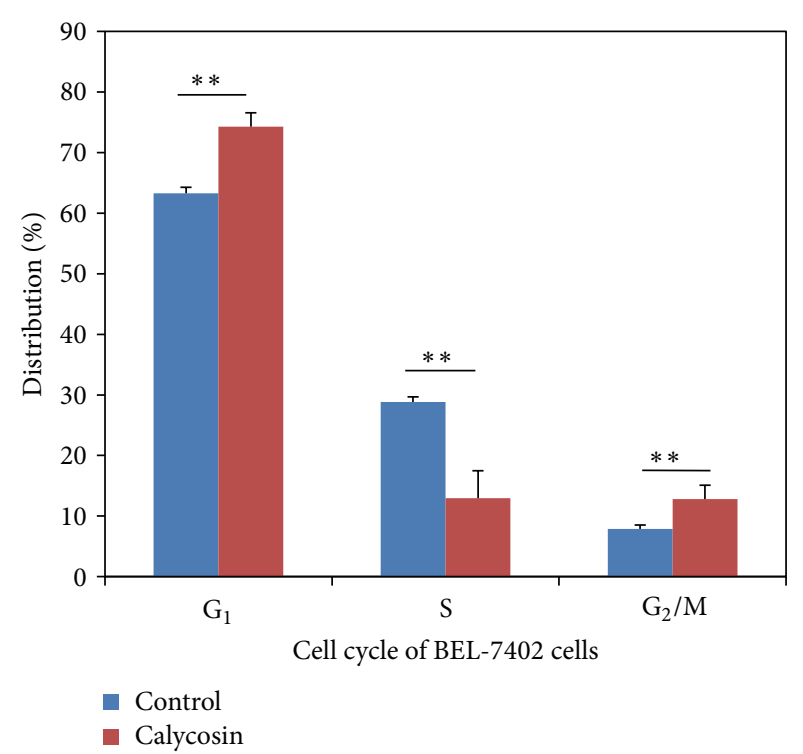

FIGURE 2: Effect of calycosin on BEL-7402 cell-cycle; ${ }^{* *}$ means that $P<0.01$ compared with control.

bands of 18S. The total RNA extracted could be used for chip hybridization. The probe marked with Cy3 (control) and Cy5 (calycosin) were hybridized with the 100 genes which were related to cell-cycle. 18 strips of differentially expressed genes were obtained (Figure 3); the ratio below 1 was regarded as downregulated gene and above 2 as upregulated gene. The upregulated and downregulated ratios of calycosin-treated/control were $3.602 \pm 1.652$ and $0.315 \pm$ 0.128 , respectively.

The data in the gene chip was normalized by the Lowess method. The differentially expressed genes were determined by the one-class method of SAM. The test repeated twice. 18 strips of differentially expressed genes was related to cell-cycle were obtained, 8 strips of expressed genes were upregulated, and 10 genes were downregulated.

3.4. Mass Spectrum Identification of Differentially Expressed Proteins. One piece of 2DE gel could be divided into approximate 1200 protein points after being analyzed by Image Master 2D Elite 5.0 software. Repeated three times each group, the matching rate of same sample was more than 95 percent. The differentially expressed points marked on $2 \mathrm{DE}$ spectrum of a graph stained with coomassie brilliant blue (Figure 4), these on the drawing of partial enlargement between calycosin treated and control. 13 proteins for upregulated expression, 2 


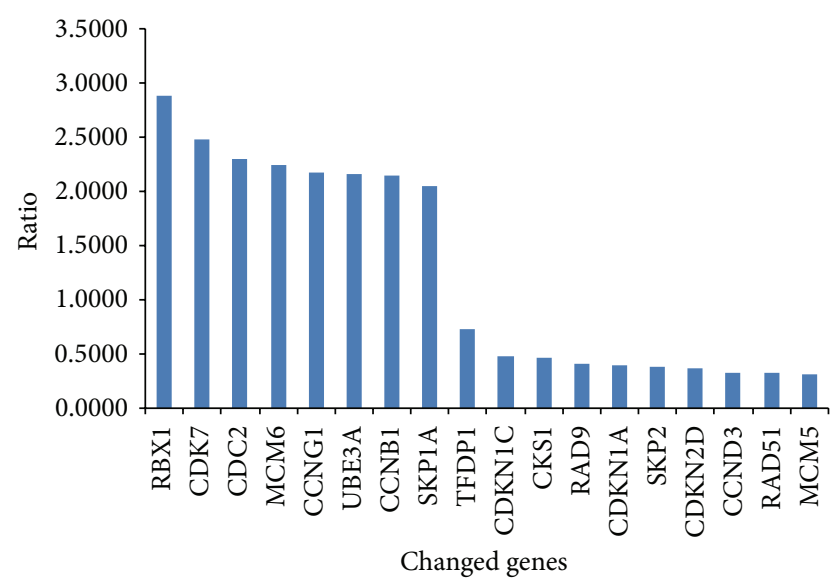

FIGURE 3: Effect of calycosin on cell-cycle genes expression of BEL7402 cell.

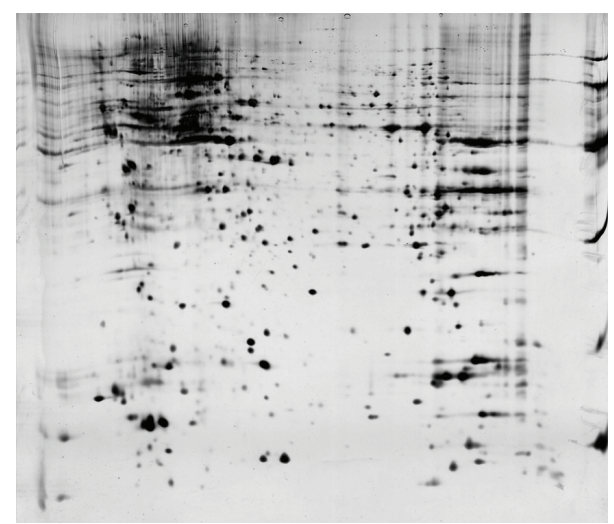

FIGURE 4: Differentially expressed proteins of human hepatoma carcinoma cells after the treatment of calycosin on 2DE gels.

proteins for downregulated expression, and 3 proteins disappearance in calycosin-treated group were shown by 2-DE gels. The $\mathrm{Mr}$ and $\mathrm{pI}$ of differentially expressed protein points were shown (Table 3).

Two-dimensional electrophoresis gel after stained was scanned using gel-image scanner and digital image document was analyzed by software for obtaining the differently expressed proteins.

14 species of proteins were identified by mass spectrum (Table 4). Sites of some proteins did not match theoretical $\mathrm{Mr}$ and $\mathrm{pI}$ because of the posttranslational modification such as glycosylation and phosphorylation. 14 species of proteins including thioredoxin Peroxidase B, keratin 1, stress-inducedphosphoprotein 1 (Hsp70/Hsp90-organizing protein) isoform 1 , abhydrolase domain containing $14 \mathrm{~B}$, pyridoxine $5^{\prime}$ phosphate oxidase, LIM and SH3 protein 1, peroxiredoxin 1, neuropolypeptide $\mathrm{h} 3$, transgelin 2 , high-mobility group box 1 , nonmetastatic cells 1 , protein (NM23A) expressed in isoform, endoplasmic reticulum protein 29 isoform 1 precursor, phosphoglycerate mutase 1 (brain), and biliverdin reductase $\mathrm{B}$ were identified by mass spectrometric detection.
TABLE 3: Isoelectric point and molecular weight of the differentially expressed proteins of human hepatoma carcinoma cells after treatment by calycosin.

\begin{tabular}{lccc}
\hline Spot & PI & Mr $(\mathrm{kD})$ & Expression \\
\hline 2 & 5.44 & 21795 & $\downarrow$ \\
8 & 9.74 & 19617 & $\downarrow$ \\
9 & 5.80 & 22708 & $\uparrow$ \\
10 & 6.77 & 28975 & $\uparrow$ \\
21 & 8.44 & 24400 & $\uparrow$ \\
22 & 7.42 & 20913 & $\uparrow$ \\
23 & 8.27 & 22096 & $\uparrow$ \\
26 & 6.41 & 18964 & - \\
31 & 6.61 & 29698 & $\uparrow$ \\
32 & 6.62 & 29969 & $\uparrow$ \\
37 & 7.81 & 68037 & $\uparrow$ \\
42 & 5.42 & 19641 & $\uparrow$ \\
52 & 6.77 & 28975 & $\uparrow$ \\
58 & 6.67 & 28786 & $\uparrow$ \\
3 & 8.15 & 65999 & $\uparrow$ \\
34 & 5.94 & 22332 & $\uparrow$ \\
61 & 7.13 & 22105 & - \\
33 & 6.62 & 29969 & \\
\hline
\end{tabular}

PI represents isoelectric point; Mr represents molecular weight; " $"$ represents the upregulated protein; " $\downarrow$ " represents the downregulated protein; "-—" represents the disappeared protein.

\section{Discussion}

In our research, calycosin, one of major monocompounds extracted from TFA, was firstly demonstrated on the inhibition on growth of BEL-7402 cells in vitro. The proliferative ratio of tumor cells mainly depended on the length of $G_{0} / G_{1}$ phase and the ability of cell division [4]. We discovered that calycosin could mainly block growth of BEL-7402 cells in $\mathrm{G}_{1}$ phase cells in $S$ phase causing to diminish markedly, after BEL-7402 cells being incubated with calycosin for 24 hours.

Flavonoids from plants can inhibit growth of tumor cell by regulating check points of cell-cycle [5-7]. Some studies had manifested that BEL-7402 cells had upregulated TFDP and E2F expression [8]. TFDP1 is a heterodimerization partner for members of the E2F family of transcription factors. E2F1/TFDP1 forms a complex involved in cell-cycle progression by the regulation of expression of cell-cycle promoters (cyclin A, cyclin E, and CDK2). TFDP-1 is one of the driver genes, which leads to higher tumor aggressiveness through deregulation of cell-cycle. Our results demonstrated that calycosin could downregulate the expression of TFDP1. In addition, abundant studies showed the high expression of SKP2 was one characteristic of human tumor growth [9-11], and SKP2 was not only used as an independent prognosis of cancer patients, but also as a new target of antitumor drugs and gene therapy [9]. In our results, calycosin affected two check points gene transcription; for example TFDP-1 and SKP2 were markedly downregulated and CDC2 and CCNB1 were upregulated expression and downregulated expression of 
TABLE 4: Differentially expressed proteins of human hepatoma carcinoma cells after treatment by calycosin.

\begin{tabular}{|c|c|c|c|c|c|}
\hline Spot & Protein name & NCB Inr ID no. & Sequence coverage (\%) & Score & Protein expression \\
\hline 2 & Thioredoxin peroxidase B & gi|9955007 & 69 & 187 & $\downarrow$ \\
\hline 3 & Keratin 1 & gi|119395750 & 50 & 159 & $\uparrow$ \\
\hline 8 & High-mobility group box 1 & gi|119628863 & 48 & 80 & $\downarrow$ \\
\hline 21 & Transgelin 2 & gi| 4507357 & 88 & 483 & $\uparrow$ \\
\hline 22 & Neuropolypeptide h3 & gi|913159 & 74 & 102 & $\uparrow$ \\
\hline 23 & Peroxiredoxin 1 & gi| 4505591 & 79 & 220 & $\uparrow$ \\
\hline 31 & LIM and SH3 protein 1 & gi|5453710 & 69 & 98 & $\uparrow$ \\
\hline 32 & Pyridoxine $5^{\prime}$-phosphate oxidase & gi|8922498 & 41 & 78 & $\uparrow$ \\
\hline 34 & Abhydrolase domain containing 14B & gi|14249382 & 58 & 233 & $\uparrow$ \\
\hline 37 & $\begin{array}{c}\text { Stress-induced phosphoprotein } 1 \\
\text { (Hsp70/Hsp90-organizing protein) } \\
\text { isoform } 1\end{array}$ & gi|114638255 & 67 & 180 & $\uparrow$ \\
\hline 42 & $\begin{array}{l}\text { Nonmetastatic cells 1, protein (NM23A) } \\
\text { expressed in isoform a }\end{array}$ & gi|38045913 & 63 & 77 & $\uparrow$ \\
\hline 52 & $\begin{array}{l}\text { Endoplasmic reticulum protein } 29 \\
\text { isoform } 1 \text { precursor }\end{array}$ & gi|5803013 & 61 & 129 & $\uparrow$ \\
\hline 58 & Phosphoglycerate mutase 1 (brain) & gi|119570326 & 61 & 135 & $\uparrow$ \\
\hline 61 & Biliverdin reductase B & gi|4502419 & 70 & 219 & $\uparrow$ \\
\hline
\end{tabular}

“ ” represents the upregulated protein; " $"$ represents the downregulated protein.

CDKN2D which may block the $\mathrm{G}_{1} / \mathrm{S}$ and $\mathrm{G}_{2} / \mathrm{M}$ transition in cancer cells.

Also we tried to investigate the mechanism of calycosin in human hepatocellular carcinoma cell by screening different proteins expression in treatment groups. We identified 12 proteins expression increasing and 2 proteins expression decreasing. The upregulated expression of transgelin 2 , pyridoxine $5^{\prime}$-phosphate oxidase, stress-induced phosphoprotein 1 (StIP1), peroxiredoxin 1, and biliverdin reductase B suggested that they played a key role in mechanism of calycosin antitumor function. Transgelin 2 protein expression is upregulated by calycosin. Previous studies show that transgelin 2 expression is decreased in some cancers. Transgelin 2 was also proved to have relationship with hepatitis B and liver carcinoma. The oncogene Ras inhibits the expression of transgelin 2 genes in thoracic wall and large bowel neoplasm and some other cancers [12-16]. The inhibition effect on tumor cell proliferation of calycosin might be caused by upregulated transgelin 2 protein expression. Calycosin can increase the PNPO protein expression. A series of researches indicated that PNPO could regulate cell proliferation and apoptosis [17], but lack in liver or neurogenic tumor [18]. Calycosin can cause stress-induced phosphoprotein 1 (StIP1) expression increase. StIP1 as a crucial chaperone has the possibility to substantial accommodation of cytogenesis in tumor, stress reaction, and cell proliferation and differentiation [19]. The expression of StIP1 is obviously downregulated in paclitaxel-resisted ovarian cancer cell strain OC3/Tax300 [20]. Calycosin can also increase two antioxidant proteins which have functions in antitumor. Peroxiredoxin, belonging to antioxidant protein superfamily involved in cell proliferation and differentiation, enhances activity of NK cell and protects free radical sensitive protein and DNA normal replication. The downregulation expression of Pdx1 was closely related to the balance between oncogene and antioncogene destroyed and the invasiveness of giant cell tumor of bone (GCTB) [21]. Biliverdin reductase B was increased by calycosin and produced bilirubin which has protection function for organism. We also found two proteins which are crucial for cancer cell growth expression inhibited by calycosin. Thioredoxin peroxidase B (Prx II) is a novel inhibitor of apoptosis of cancer cells [22]. They efficiently reduced the intracellular level of $\mathrm{H}_{2} \mathrm{O}_{2}$ produced stimulated by various cell surface ligands. The peroxiredoxins family was reported to be closely related to various causes of liver fibrosis. They were found to be upregulated in liver fibrosis caused by chemical induction. Prx II has shown a significant upregulation at the middle-stage fibrosis and played an important role in hepatocarcinogenesis [23]. Research showed that the hepatocellular carcinoma cells proliferation and clone formation decreased and cell apoptosis was enhanced obviously when Prx II expression was inhibited [24]. Prx II also has shown a downregulation in the hepatocellular carcinoma cells after being treated with calycosin, which might be related to the antitumor mechanism of calycosin. We found that calycosin can inhibit thioredoxin peroxidase B protein expression. High-mobility group box 1 protein (HMGB1), a chromatin-associated nuclear protein and extracellulardamage-associated molecular pattern molecule (DAMP), is an evolutionarily ancient and critical regulator of cell death and survival. Over-expression of HMGB1 is associated with each of the hallmarks of cancer including unlimited replicative potential, angiogenesis, evasion of apoptosis, selfsufficiency in growth signals, and insensitivity to inhibitors of growth. In our study, calycosin downregulated HMGB1, 
which may be related to the antitumor mechanism of calycosin on hepatocellular carcinoma cells.

In conclusion, calycosin can inhibit cell growth of BEL7402 by blocking cell-cycle and regulating some tumor genes translation. The cell-cycle-related genes which we found only in RNA array chip but not in the 2D gel maybe because the expression quantity is limited. The molecular mechanism was related to downregulated expression of TFDP-1, SKP2, and CDKN2D and upregulated expression of CDC2, CDK7, and CCNB1. And then we discovered certain proteins related to antitumor including transgelin 2, PNPO, StIP1, PDX 1, biliverdin reductase B, HMGB1, and Prx II which were changed after calycosin treatment. The functions of differentially expressed proteins may have a possibility to be involved in antitumor mechanism of calycosin.

\section{Conflict of Interests}

The authors declare that they have no financial and personal relationships with other people or organizations that can inappropriately influence their work. All the authors declare that they have no competing interests.

\section{Authors' Contribution}

Dongqing Zhang and Shufang Wang contributed equally to this work.

\section{Acknowledgment}

This research was supported by a Grant from the National Nature Foundation of China (no. 30572349).

\section{References}

[1] D. Q. Wang, Y. Li, Y. P. Tian et al., "Inhibition effects of total flavonoids of astragalus on BEL-7402 cell in vitro," Academic Journal of PLA Postgraduate Medical School, vol. 26, no. 5, pp. 331-333, 2005.

[2] D. Q. Zhang, Y. Zhuang, J. Pan et al., "Investigation of effects and mechanisms of total flavonoids of astragalus and calycosin on human erythroleukemia cells," Oxidative Medicine and Cellular Longevity, vol. 2012, 5 pages, 2012.

[3] D.-J. Xu, Q. Wu, Y. Yang, and M.-Z. Chen, "Antitumor activity of AST and its mechanism of action," Chinese Pharmacological Bulletin, vol. 19, no. 7, pp. 823-826, 2003.

[4] A. Deshpande, P. Sicinski, and P. W. Hinds, "Cyclins and cdks in development and cancer: a perspective," Oncogene, vol. 24, no. 17, pp. 2909-2915, 2005.

[5] L. A. Beltz, D. K. Bayer, A. L. Moss, and I. M. Simet, "Mechanisms of cancer prevention by green and black tea polyphenols," Anti-Cancer Agents in Medicinal Chemistry, vol. 6, no. 5, pp. 389-406, 2006.

[6] S. M. Meeran and S. K. Katiyar, "Cell cycle control as a basis for cancer chemoprevention through dietary agents," Frontiers in Bioscience, vol. 13, no. 6, pp. 2191-2202, 2008.

[7] T. Walle, "Methoxylated flavones, a superior cancer chemopreventive flavonoid subclass?" Seminars in Cancer Biology, vol. 17, no. 5, pp. 354-362, 2007.
[8] L.-X. Liu, H.-C. Jiang, Z.-H. Liu et al., "Gene expression profiles of hepatoma cell line BEL-7402," Hepato-Gastroenterology, vol. 50, no. 53, pp. 1496-1501, 2003.

[9] D. D. Hershko, "Oncogenic properties and prognostic implications of the ubiquitin ligase Skp2 in cancer," Cancer, vol. 112, no. 7, pp. 1415-1424, 2008.

[10] G. Rodier, P. Coulombe, P.-L. Tanguay, C. Boutonnet, and S. Meloche, "Phosphorylation of Skp2 regulated by CDK2 and Cdc14B protects it from degradation by APCCdh1 in G1 phase," EMBO Journal, vol. 27, no. 4, pp. 679-691, 2008.

[11] M. S. Song, S. J. Song, S. J. Kim, K. Nakayama, K. I. Nakayama, and D.-S. Lim, "Skp2 regulates the antiproliferative function of the tumor suppressor RASSF1A via ubiquitin-mediated degradation at the G1-S transition," Oncogene, vol. 27, no. 22, pp. 3176-3185, 2008.

[12] J. M. Shields, K. Rogers-Graham, and C. J. Der, "Loss of transgelin in breast and colon tumors and in RIE-1 cells by Ras deregulation of gene expression through Raf-independent pathways," Journal of Biological Chemistry, vol. 277, no. 12, pp. 9790-9799, 2002.

[13] D. Lawson, M. Harrison, and C. Shapland, "Fibroblast transgelin and smooth muscle SM22alpha are the same protein, the expression of which is down-regulated in many cell lines," Cell Motility and the Cytoskeleton, vol. 38, pp. 250-257, 1997.

[14] R. K. Prinjha, C. E. Shapland, J. J. Hsuan, N. F. Totty, I. J. Mason, and D. Lawson, "Cloning and sequencing of cDNAs encoding the actin cross-linking protein transgelin defines a new family of actin-associated proteins," Cell Motility and the Cytoskeleton, vol. 28, no. 3, pp. 243-255, 1994.

[15] C. Shapland, J. J. Hsuan, N. F. Totty, and D. Lawson, "Purification and properties of transgelin: a transformation and shape change sensitive actin-gelling protein," Journal of Cell Biology, vol. 121, no. 5, pp. 1065-1073, 1993.

[16] Z. Yang, Y.-J. Chang, H. Miyamoto et al., “Transgelin functions as a suppressor via inhibition of ARA54-enhanced androgen receptor transactivation and prostate cancer cell growth," Molecular Endocrinology, vol. 21, no. 2, pp. 343-358, 2007.

[17] J.-F. Léonard, M. Courcol, C. Mariet et al., "Proteomic characterization of the effects of clofibrate on protein expression in rat liver," Proteomics, vol. 6, no. 6, pp. 1915-1933, 2006.

[18] E. O. Ngo, G. R. LePage, J. W. Thanassi, N. Meisler, and L. M. Nutter, "Absence of pyridoxine- 5 ' - phosphate oxidase (PNPO) activity in neoplastic cells: isolation, characterization, and expression of PNPO cDNA," Biochemistry, vol. 37, no. 21, pp. 7741-7748, 1998.

[19] S. Bagci, J. Zschocke, G. F. Hoffmann et al., "Pyridoxal phosphate-dependent neonatal epileptic encephalopathy," Archives of Disease in Childhood, vol. 93, no. 2, pp. F151-F152, 2008.

[20] W. J. Zhang, H. X. Li, L. Song et al., "Screening differential expression of Taxo-1 resistance related gene of ovarian carcinnoma by cDNA microarray," Cancer Research on Prevention and Treatment, vol. 33, no. 1, pp. 32-35, 2006.

[21] Y.-Z. Li, Z.-L. Gao, J.-L. Zuo et al., "Expressions of annexinal, 14-3-3 protein $\varepsilon$ and peroxiredoxin 1 giant cell tumor of bone," Journal of Jilin University, vol. 32, no. 3, pp. 489-492, 2006.

[22] B. Husbeck, M. I. Berggren, and G. Powis, "DNA microarray reveals increased expression of thioredoxin peroxidase in thioredoxin-1 transfected cells and its functional consequences," Advances in Experimental Medicine and Biology, vol. 500, pp. 157-168, 2001.

[23] Y. Lu, J. Liu, C. Lin et al., "Peroxiredoxin 2: a potential biomarker for early diagnosis of Hepatitis B Virus related liver fibrosis 
identified by proteomic analysis of the plasma," BMC Gastroenterology, vol. 10, article 115, 2010.

[24] Y. Li, X. Qin, J. Cui et al., "Proteoma analysis of aflatioxin B1induced hepatocarcino genesis in the tree shrew and functional identification of candidate protein peroxiredoxin?" Proteomics, vol. 8, no. 7, pp. 1490-1501, 2008.

[25] M. E. Minard, L. M. Ellis, and G. E. Gallick, "Tiam1 regulates cell adhesion, migration and apoptosis in colon tumor cells," Clinical and Experimental Metastasis, vol. 23, no. 5-6, pp. 301-313, 2006. 


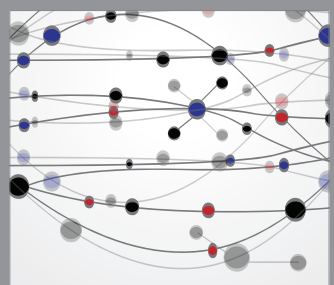

The Scientific World Journal
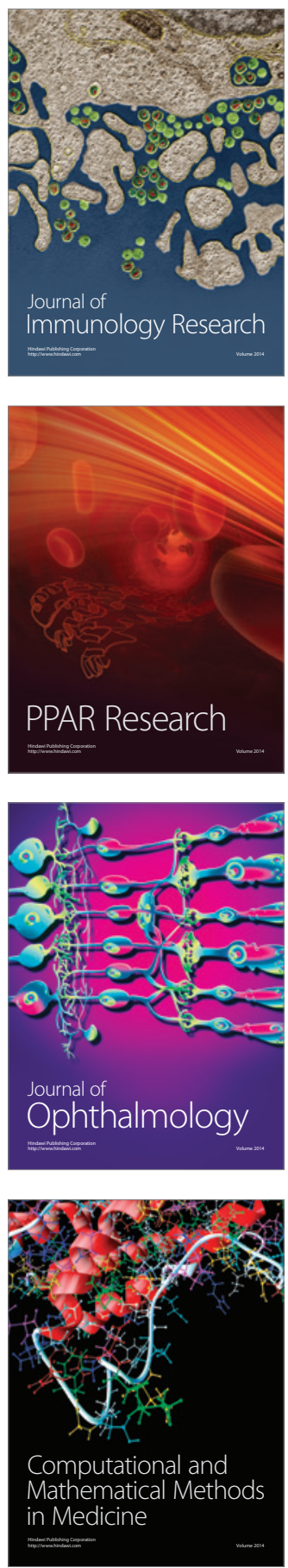

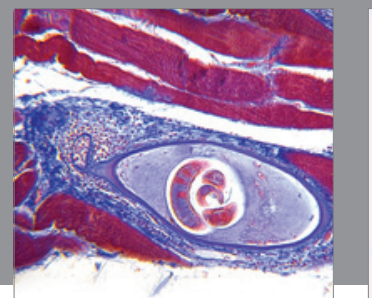

Gastroenterology

Research and Practice
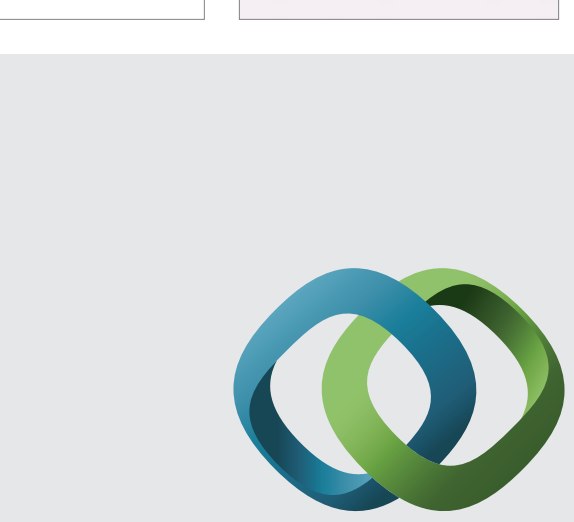

\section{Hindawi}

Submit your manuscripts at

http://www.hindawi.com
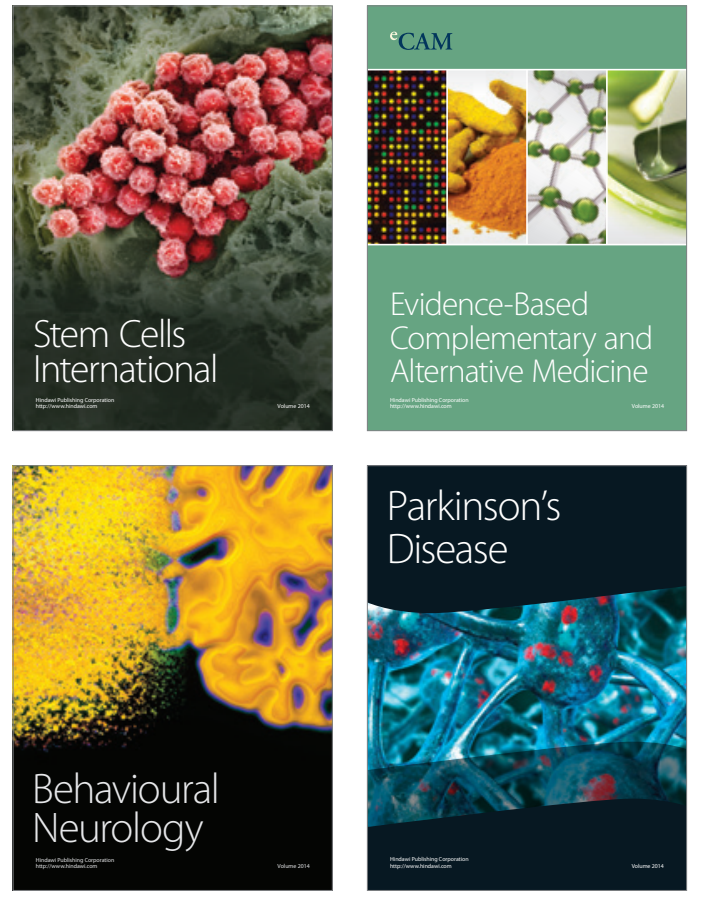
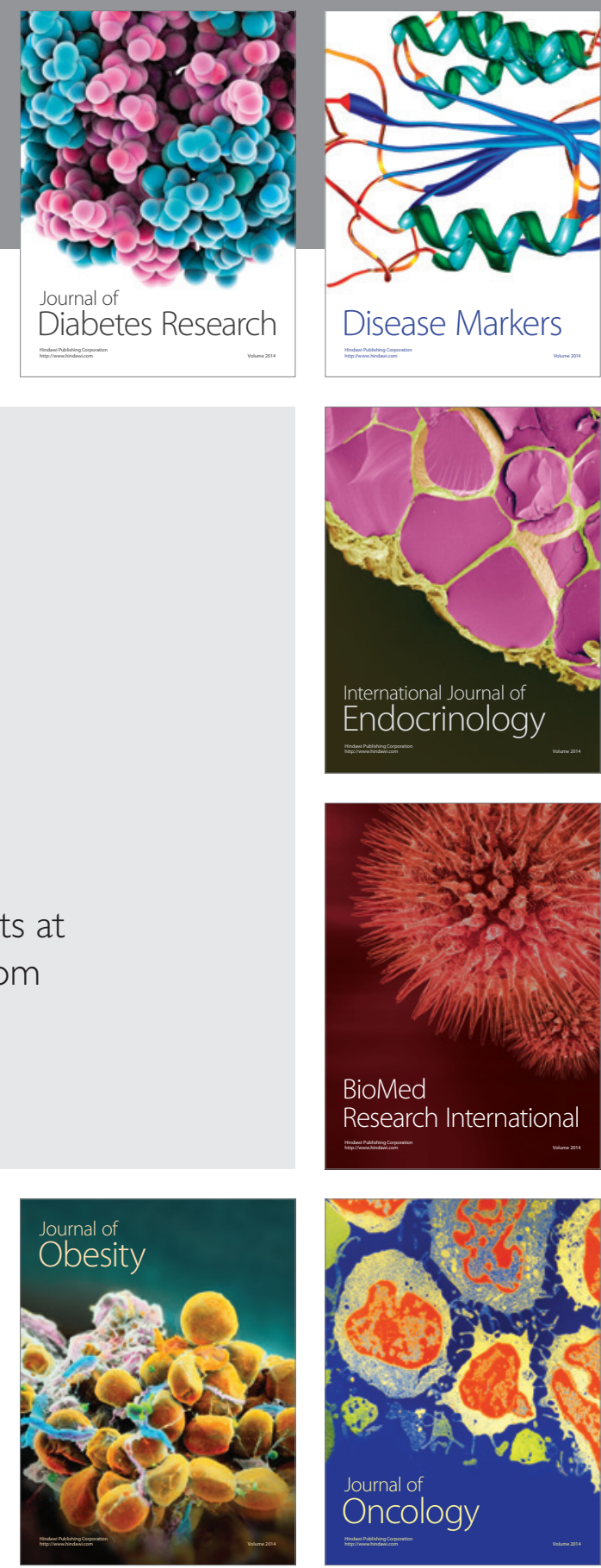

Disease Markers
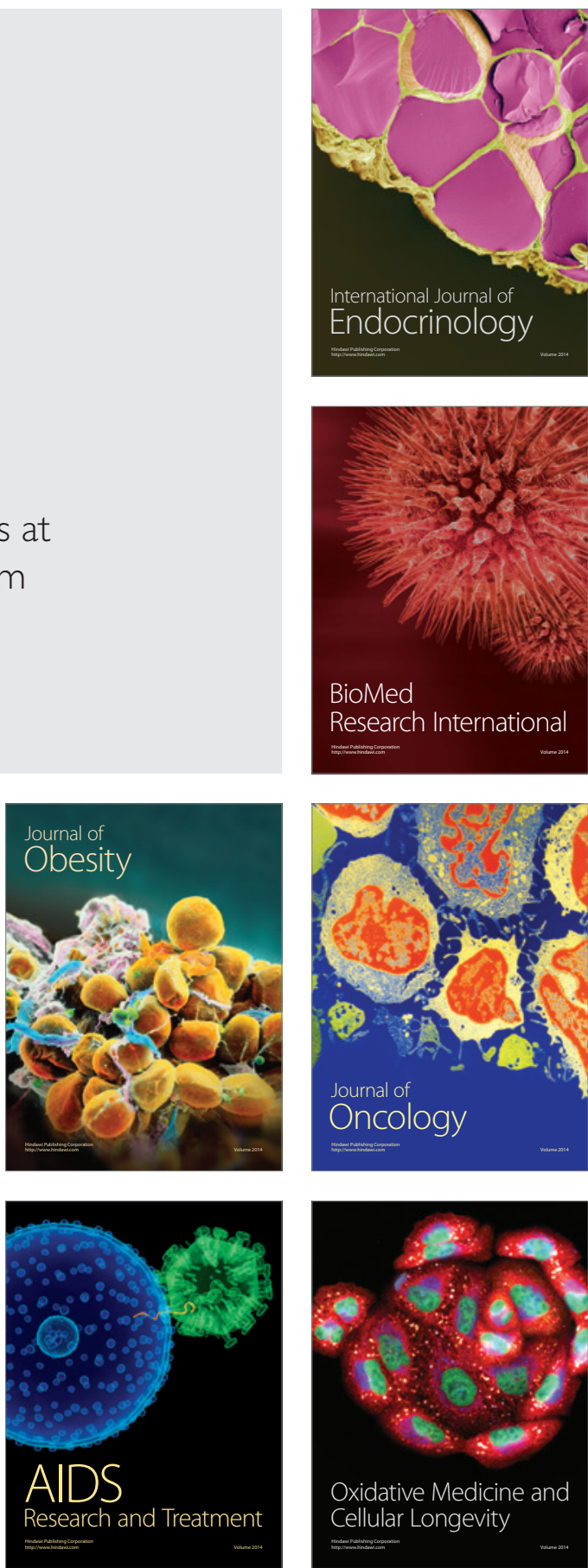